Article

\title{
Genome-Wide Association Study of Body Conformation Traits by Whole Genome Sequencing in Dazu Black Goats
}

\author{
Bowen Gu ${ }^{1,2,3}$, Ruifan Sun ${ }^{1,2,3}$, Xingqiang Fang ${ }^{1,2,3}$, Jipan Zhang ${ }^{1,2,3}{ }^{(0)}$, Zhongquan Zhao ${ }^{1,2,3}$, Deli Huang ${ }^{4}$, \\ Yuanping Zhao ${ }^{5}$ and Yongju Zhao ${ }^{1,2,3, *(\mathbb{D})}$
}

1 College of Animal Science and Technology, Southwest University, Chongqing 400715, China; gubw04@163.com (B.G.); sunruifan111@163.com (R.S.); 15881044561@163.com (X.F.); jpanzhang@live.com (J.Z.); zhaozhongquan@swu.edu.cn (Z.Z.)

2 Chongqing Key Laboratory of Herbivore Science, Chongqing 400715, China

3 Chongqing Engineering Research Center for Herbivores Resource Protection and Utilization, Chongqing 400715, China

4 Tengda Animal Husbandry Co., Ltd., Chongqing 402360, China; huangdl116@163.com

5 Dazu County Agriculture and Rural Committee, Chongqing 402360, China; dzxmz@163.com

* Correspondence: zyongju@163.com

Citation: Gu, B.; Sun, R.; Fang, X.; Zhang, J.; Zhao, Z.; Huang, D.; Zhao, Y.; Zhao, Y. Genome-Wide Association Study of Body Conformation Traits by Whole Genome Sequencing in Dazu Black Goats. Animals 2022, 12, 548. https:// doi.org/10.3390/ani12050548

Academic Editor: Michael E. Davis

Received: 13 December 2021

Accepted: 19 February 2022

Published: 23 February 2022

Publisher's Note: MDPI stays neutral with regard to jurisdictional claims in published maps and institutional affiliations.

Copyright: (C) 2022 by the authors. Licensee MDPI, Basel, Switzerland. This article is an open access article distributed under the terms and conditions of the Creative Commons Attribution (CC BY) license (https:// creativecommons.org/licenses/by/ $4.0 /)$.
Simple Summary: Body conformation traits are economically important in the goat meat industry. Good growth performance in goats, including an accelerated growth rate, can improve carcass weight and meat yield. The identification of genetic variants associated with these traits provides a basis for the genetic improvement of growth performance. In this study, we measured six body conformation traits, including body height, body length, cannon circumference, chest depth, chest width, and heart girth. By a genome-wide association study of a Chinese meat goat breed, 53 significant single nucleotide polymorphisms and 42 candidate genes associated with these traits were detected. These findings improve our understanding of the genetic basis of body conformation traits in goats.

Abstract: Identifying associations between genetic markers and economic traits has practical benefits for the meat goat industry. To better understand the genomic regions and biological pathways contributing to body conformation traits of meat goats, a genome-wide association study was performed using Dazu black goats (DBGs), a Chinese indigenous goat breed. In particular, 150 DBGs were genotyped by whole-genome sequencing, and six body conformation traits, including body height (BH), body length (BL), cannon circumference (CC), chest depth (CD), chest width (CW), and heart girth (HG), were examined. In total, 53 potential SNPs were associated with these body conformation traits. A bioinformatics analysis was performed to evaluate the genes located close to the significant SNPs. Finally, 42 candidate genes (e.g., PSTPIP2, C7orf57, CCL19, FGF9, SGCG, FIGN, and $S I P A 1 L$ ) were identified as components of the genetic architecture underlying body conformation traits. Our results provide useful biological information for the improvement of growth performance and have practical applications for genomic selection in goats.

Keywords: body conformation traits; whole genome sequencing; genome-wide association study; single nucleotide polymorphism; goat

\section{Introduction}

As one of the oldest domesticated livestock, goats provide abundant meat, milk, and fiber [1]. Most of the world's goat population is found in South-East Asia and Africa, where goats are the major source of meat production [2]. Owing to the rapid growth of the world's population and increased demand for diversified meat, the goat meat industry has become increasingly important [3]. The history of human migration shaped the genetic structure of goats. One-third of indigenous goat breeds with unique biological characteristics live in Southwest China [4]. Today, there are nearly 120,000 Dazu black goats (DBGs), which are 
raised in Dazu County of Chongqing (267-934 m above sea level, Southwest China) and are mostly used for meat production [5]. Therefore, the development of candidate genetic markers and functional genes for the optimization of breeding has important practical implications for the goat meat industry.

Growth performance is an economic trait in the meat goat industry. Body conformation traits of goats directly reflect the body size, structure, and development, which are closely related to physiological function and production performance. Body height $(\mathrm{BH})$, body length (BL), heart girth (HG), chest width (CW), chest depth (CD), and cannon circumference $(\mathrm{CC})$ are the most frequently used body conformation traits [6]. However, these traits are generally polygenic and are influenced by environmental factors, making association mapping difficult [7]. Recently, bioinformatics approaches have been applied to study the genetic basis of body conformation traits. The PRDM6 gene influences growthrelated traits, such as $\mathrm{CC}, \mathrm{CD}$, and $\mathrm{CW}$, in the early growth and development of goats [8]. PITX2 is significantly associated with body height and body length in Guanzhong dairy goats and Hainan black goats [9]. Two indels in PRLR are significantly correlated with BL, $\mathrm{BH}, \mathrm{CD}, \mathrm{HG}$, and CC [10].

Genome-wide association studies (GWAS) are a popular approach to detect genomewide single-nucleotide polymorphisms (SNPs) related to phenotypic traits [11]. To date, GWAS have been widely applied to the goat litter size [12,13], horn status [14], coat color [15], and milk quality [16]. In a study of $4840 \mathrm{New}$ Zealand dairy goats, 43 significant SNPs were associated with fat, proteins, and somatic cell scores [17]. Wang et al. used whole-genome resequencing (WGS) to detect 12 candidate genes from two groups of goats with different litter sizes [18]. Compared to low-density SNP chips, WGS can improve the accuracy and power of GWAS for the detection of SNPs associated with complex traits $[19,20]$. As the cost of WGS is rapidly decreasing, GWAS using genotypes from WGS have been reported in cattle [21], pigs [22], and goats [23]. In this study, we performed WGS of 150 DBGs and conducted GWAS of six body conformation traits to identify the significant SNPs and related candidate genes.

\section{Materials and Methods}

\subsection{Ethics Statement}

The Chongqing Key Laboratory of Forage \& Herbivore approved this experiment. All goat experiments followed the Southwest University Institutional Animal Care and Use Committee regulations (2019, No. GB14925-2010), and no animals were anesthetized or euthanized during the study.

\subsection{Animals, Phenotypes, and DNA Extraction}

A total of 150 female DBGs weighing 35-40 kg from Tengda Farm (Chongqing, China) were used in this study. According to the China National Standard (NY/T 1236-2006) (Supplementary Materials Figure S1), our team had a specific person to keep the goat calm, a specific person to measure its body conformation traits, another specific person to record the data, and others to calm the other goats. Then, six body conformation traits, (i.e., $\mathrm{BH}, \mathrm{BL}$, CC, CD, CW, and HG) of 150 goats were determined (Supplementary Materials Table S1). The pedigree information for each goat could be traced back at least three generations. We ensured that there was no direct kinship between these goats. Five milliliters of blood were collected from every individual and brought back to the laboratory using an ice box. In the laboratory, these samples were stored at $-80^{\circ} \mathrm{C}$. Next, the standard phenol-chloroform protocol was used to extract the DNA from all blood samples.

\subsection{Whole-Genome Sequencing}

The integrity and concentration of the genomic DNA were assessed by agarose gel electrophoresis and a NanoDrop spectrophotometer (Thermo Fisher Scientific, Waltham, MA, USA), respectively. Then, the sequencing of each sample was performed on the DNBSEQ-T7 platform (Complete Genomics and MGI Tech, Shenzhen, China). According to 
the standard protocol, the DNA samples were tested, amplified, and purified, and libraries were constructed. During library construction, the genomic DNA was randomly broken into fragments of about $350 \mathrm{bp}$ by the fragmentation instrument. After end repair, the sequencing connectors were added for sequencing. Since the raw data contained reads with junctions or low quality, filtering was performed as follows: (1) reads with adapters were removed, (2) paired reads were removed when the $\mathrm{N}$ content exceeded $1 \%$ of bases in a read, and (3) paired reads were removed when the quality score $\leq 5$.

\subsection{Quality Control}

Clean data were mapped to the goat genome (ARS1) [24] using Burrows-Wheeler Aligner (BWA) (version 0.7.15) [25]. Then, Picard (v 1.129, http:/ / broadinstitute.github.io/ picard, accessed on 24 February 2015) and Samtools (v 1.10, https:/ /github.com/samtools/ samtools, accessed on 7 December 2019) [25] were used to sort the BAM files. SNPs were identified for all samples using the GATK Unified Genotyper (version 3.4.46) [26]. The detection process was as follows: (1) comparison with the reference genome and de-duplication; (2) the generation of SNP and indel variant files; (3) filtering of the SNPs and indels based on Quality by Depth $(\mathrm{QD})<2.0$, root mean square of Mapping Quality $(\mathrm{MQ})<40.0$, Fisher Strand (FS) > 60.0, HaplotypeScore > 13.0, and MQRankSum $\leq 12.5$; and (4) filtering SNP clusters (i.e., multiple SNPs within $5 \mathrm{bp}$ ), SNPs near indels (i.e., within $5 \mathrm{bp}$ ), adjacent indels (i.e., indels separated by $<10 \mathrm{bp}$ ), and loci with GQ (Genotype Quality) less than 20. Then, the following quality control criteria were implemented for the variants detected and individuals: (1) $>10 \%$ deletions, (2) minor allele frequency (MAF) $<1 \%$, and (3) Hardy-Weinberg equilibrium (HWE) testing with $p<1 \times 10^{-6}$. After quality control, all individuals and 16,776,605 SNPs were retained for subsequent analyses.

\subsection{Population Structure Analysis}

A principal components analysis (PCA) was applied to estimate the population structure of 150 goats using the FactoMineR package in R (v4.0.4). The top PCs explaining the largest proportion of variance were extracted to draw scatter plots and to evaluate the population structure [27]. Heat maps were plotted against the kinship matrix to visualize the level of relatedness among individuals within the goat population.

\subsection{Genome-Wide Association Studies}

Since GWAS does not allow missing or unknown SNPs, we used Beagle 5.0 [28] to infer missing data. The mixed linear model used in this study can effectively correct for population structure and complex kinship relationships within the population [29]:

$$
y=\mathbf{X} \boldsymbol{\beta}+\mathbf{Z}_{\mathbf{k}} \gamma_{\mathbf{k}}+\xi+e
$$

where $y$ is a phenotype, $\mathbf{X} \boldsymbol{\beta}$ is the population structure effect and fixed effects, such as year and season, $\mathbf{Z}_{\mathbf{k}} \gamma_{\mathbf{k}}$ is the marker effect to be tested; $\xi \sim N\left(0, K \phi^{2}\right)$ is the polygenic effect, $e \sim N$ $\left(0, I \sigma^{2}\right)$ is the residual effect, and $\mathrm{K}$ is the kinship matrix inferred from the SNPs. Then, we used GEMMA [30] to obtain SNPs significantly associated with body conformation traits. The Bonferroni-corrected $p$-value threshold was $6.01 \times 10^{-8}$, with a corresponding $-\log p$-value of 7.22. Significant SNPs were visualized by Manhattan plots, and $p$-value distributions (expected vs. observed $p$-values on a $-\log _{10}$ scale) were visualized by a quantile-quantile $(\mathrm{QQ})$ plot.

\subsection{Functional Enrichment Analysis}

Candidate genes were identified according to their physical positions and functions based on the ARS1 reference genome assembly. Significant SNPs were annotated to their nearest genes using ANNOVAR. Gene Ontology (GO) is an international standardized gene function classification system based on GO terms in three broad categories: molecular function (MF), cellular component (CC), and biological process (BP). Kyoto Encyclopedia of Genes and Genomes (KEGG) is the main public database to identify pathways that are 
significantly enriched for candidate genes compared to reference genes. Gene Ontology (GO) and KEGG pathway analyses of the candidate genes were performed using the clusterProfiler package in R (v4.0.4) [31].

\section{Results}

\subsection{Phenotypic Data Analysis}

The descriptive statistics for six body conformation traits $(\mathrm{BH}, \mathrm{BL}, \mathrm{CC}, \mathrm{CD}, \mathrm{CW}$, and HG) are presented in Table 1. To ensure the reliability of the results for follow-up analyses, we estimated the standard deviations, confidence intervals, and coefficients of variation. The dependent variables (BH, BL, etc.) were approximately normally distributed (Supplementary Materials Figure S2).

Table 1. Descriptive statistics of the body conformation traits; $n=150$.

\begin{tabular}{ccccccc}
\hline Trait & Max $(\mathbf{c m})$ & Min $(\mathbf{c m})$ & Mean $(\mathbf{c m})$ & Var & Std. Dev & CV \\
\hline BH & 75.5 & 50.4 & 63.52 & 12.70 & 3.56 & $5.6 \%$ \\
BL & 78.5 & 54.3 & 67.27 & 79.16 & 4.38 & $6.5 \%$ \\
CC & 10.3 & 6.8 & 8.13 & 0.33 & 0.58 & $7.0 \%$ \\
CD & 38.3 & 20.5 & 29.78 & 6.06 & 2.46 & $8.2 \%$ \\
CW & 23.9 & 8.2 & 17.13 & 4.68 & 2.16 & $12.0 \%$ \\
HG & 99.5 & 68.7 & 81.65 & 29.60 & 5.44 & $6.0 \%$ \\
\hline
\end{tabular}

BH: Body Height; BL: Body Length; CC: Cannon Circumference; CD: Chest depth; CW: Chest Width; HG: Heart Girth; Var: variance; Std.dev: Standard Deviation; CV: Coefficient of Variation.

\subsection{Genotypic Data}

After library construction and sequencing with the DNBSEQ-T7 system, $17.83 \mathrm{~GB}$ of raw data were produced for each individual. After the filtering processes, sequence data for 150 DBGs were mapped to the reference genome to a mean depth of $6 \times$, with mapping rates of $99.06 \%$ on average (Table 2). A total of $25,269,456$ SNPs and 2,940,245 small indels were in the whole dataset. There were 17,286,575 transitions and 8,127,462 transversions, leading to a Ti/Tv ratio of 2.13 (Supplementary Materials Table S2). The SNP distribution in the goat chromosomes is shown in Figure 1A. After quality control, 16,776,605 SNPs from 150 animals were retained for further analysis.

Table 2. Summary of the whole-genome sequencing data.

\begin{tabular}{cccccc}
\hline Goat (n) & ${\text { Raw Bases }(\mathbf{G b}){ }^{\mathbf{1}}}$ & Reads Mapped to the Reference $^{\mathbf{1}}$ & Total Reads $^{\mathbf{1}}$ & Mapping Ratio (\%) $^{\mathbf{1}}$ & Coverage Depth $^{\mathbf{1}}$ \\
\hline 150 & 17.83 & 116293649 & 117573225 & 99.06 & 6.00 \\
\hline \multicolumn{5}{c}{ Average per individual. }
\end{tabular}

\subsection{Population Structure Analysis}

The population structure was evaluated based on the top three PCs by 3D plots (Figure 1B). In the PCA, three PCs explained $6.45 \%$ of the total variance, including $2.32 \%, 2.12 \%$, and $2.01 \%$. The results indicated that not all individuals clustered together and suggested there was genetic drift between some DBGs and other breeds of goats. Supplementary Materials Figure S3 shows a kinship heat map. To avoid false positives caused by population stratification, PCs and a kinship matrix were used as covariates in the fixed effects model for an association analysis. 

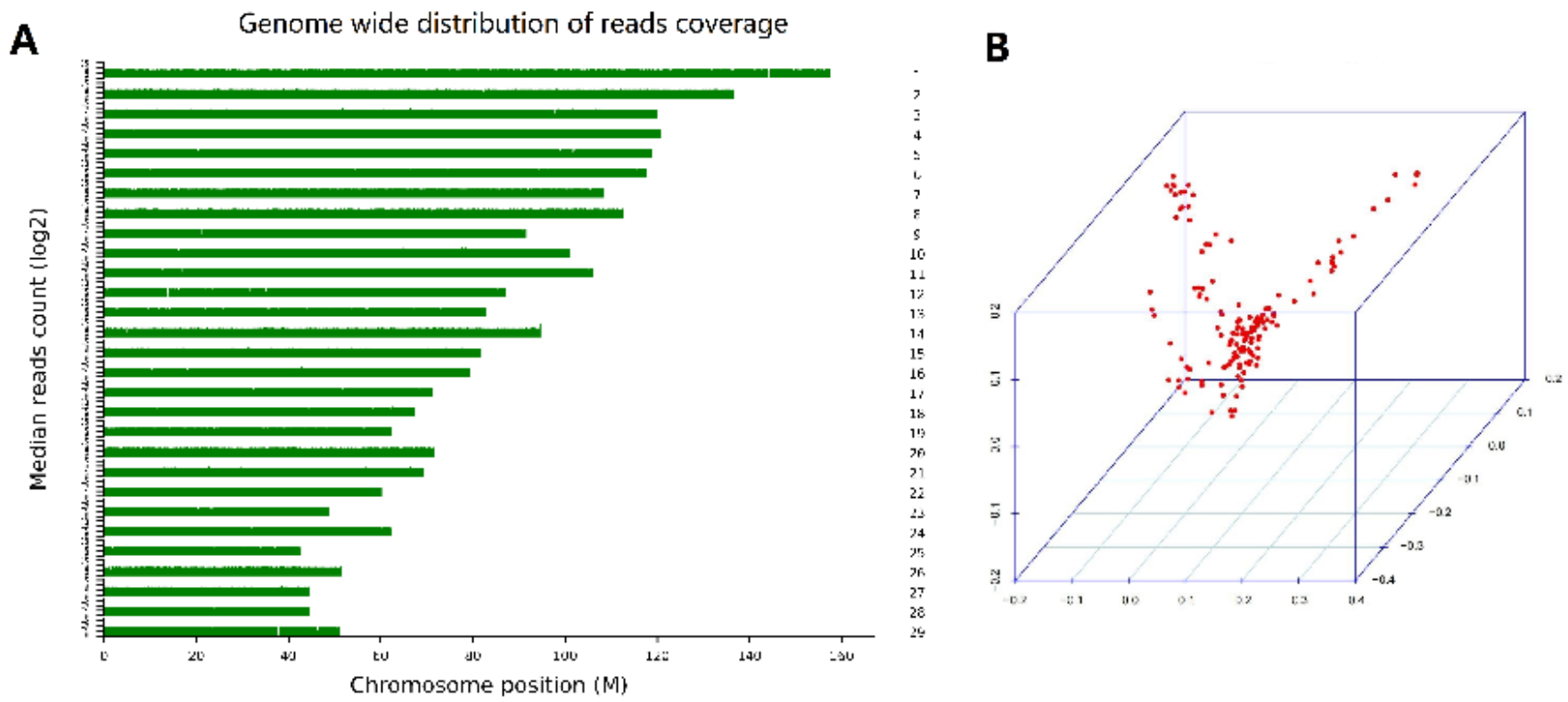

Figure 1. Read density distribution on 29 chromosomes. (A) Population structure plots based on 150 goats. The first three principal components (PCs) were used to display the population structure by a $3 \mathrm{D}$ plot $(\mathbf{B})$.

\subsection{Genome-Wide Association Study}

The QQ plot in Figure 2 shows the observed and expected $p$-values from the GWAS. Most SNPs did not deviate from the expected $p$-values, suggesting that the models for GWAS were reasonable. After filtering and obtaining adjusted phenotypes, GWAS was performed on the SNPs. Based on stringent thresholds, we found 53 genome-wide significant SNPs (corrected $p$-value $<5.96 \times 10^{-8}$ ) for BH, BL, CC, and CD and no significant SNPs for CW and HG (Figure 3). Some candidate genes corresponding to the SNPs were identified. The gene corresponding to the significant SNP associated with $\mathrm{BH}$ was ESCHIG00000017110, located on chromosome 27. For BL, PSTPIP2 corresponded to the significant SNP located on chromosome 24. We identified 28 genes corresponding to 40 SNPs linked to CC, mainly located on chromosome 8 (UBAP1, CNTFR, RPP25L, CCL19, CCL21, TLR4, BRINP1, and CDK5RAP2) and chromosome 12 (FGF9 and SGCG). Additionally, 11 significant SNPs associated with CD were located on chromosomes 4, 20, and 22. The genes nearest to these SNPs were SUN3, C7orf57, MANEA, ENSCHIG00000020259, DOK5, CBLN4, ENSCHIG00000022146, CDH9, ENSCHIG00000008494, CDKAL1, SOX4, and EDEM1. (Supplementary Materials Table S3).

\subsection{GO Enrichment and KEGG Analysis}

The candidate genes were significantly enriched for several GO terms and KEGG pathways. The top significant GO terms were "GO: 1903319, positive regulation of protein maturation", "GO: 0048144, fibroblast proliferation", and other growth-associated GO terms (Supplementary Materials Table S4). The KEGG pathway analysis revealed that these candidate genes were highly enriched in four pathways. These pathways included NF-K B signaling, viral protein interaction with cytokine and cytokine receptors, cytokine-cytokine receptor interactions, and protein processing in the endoplasmic reticulum (Supplementary Materials Table S5). 

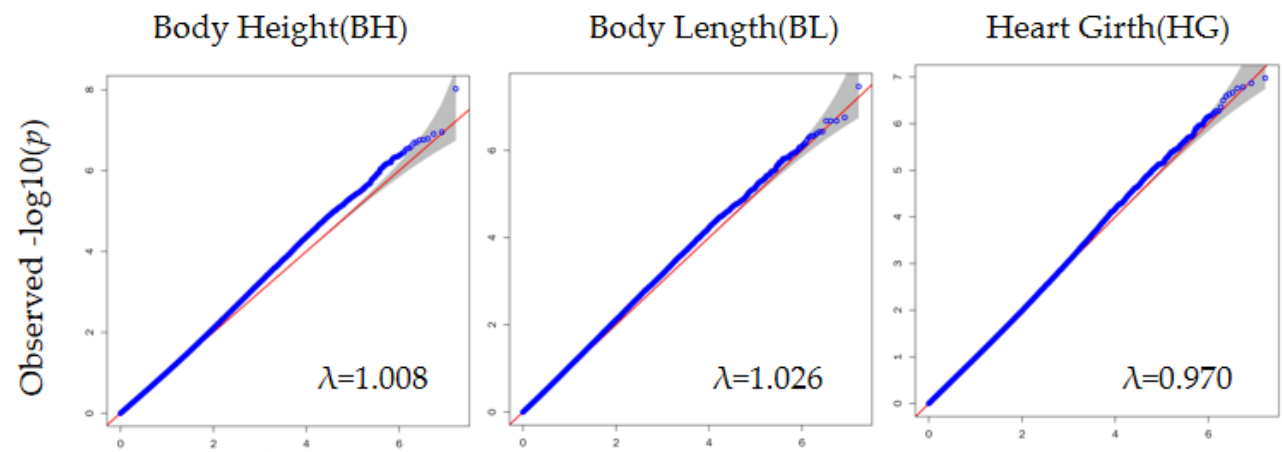

Cannon Circumference $(\mathrm{CC})$
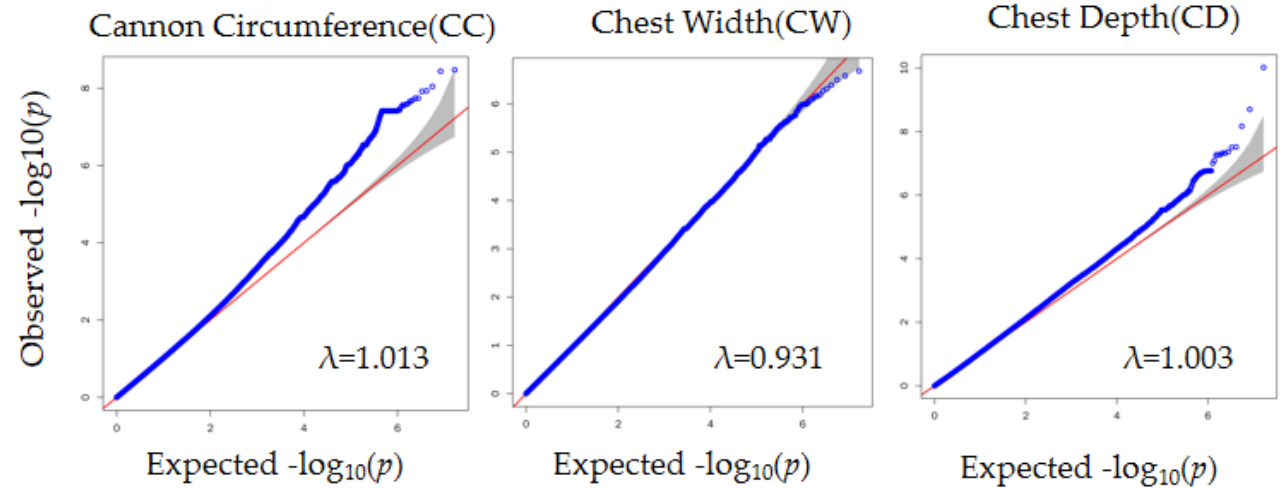

Figure 2. Quantile-quantile (QQ) plots of the six traits (BH, BL, HG, CC, CW, and CD) drawn by the expected $p$-value (the uniformly distributed quantile from 0 to 1) and observed $p$-value for each SNP. The shaded parts are the confidence intervals. $\lambda$ : genomic inflation factor.

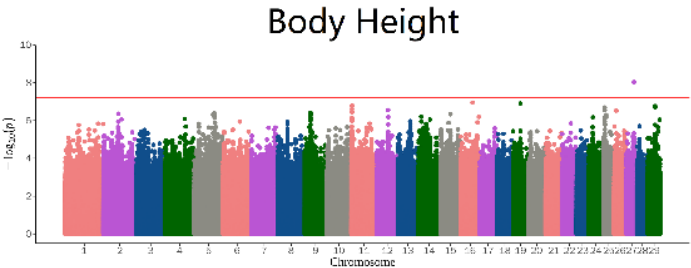

A

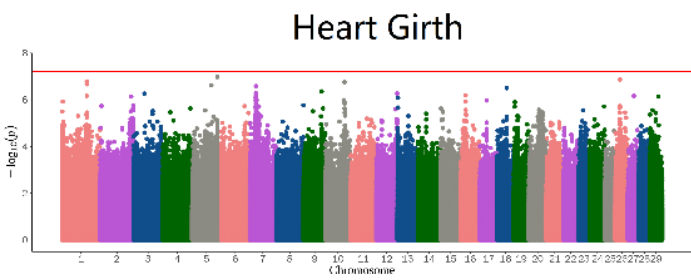

C

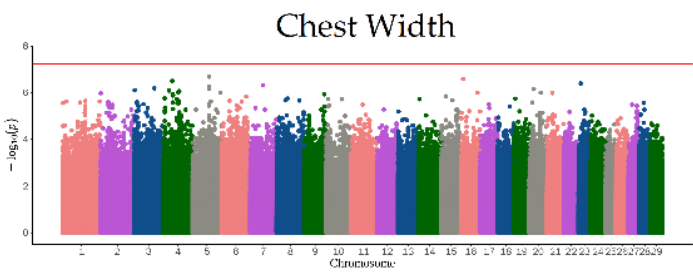

E

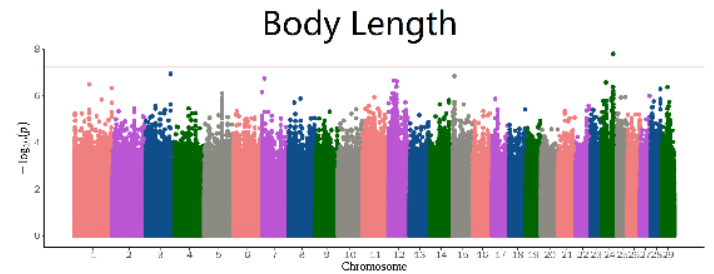

B

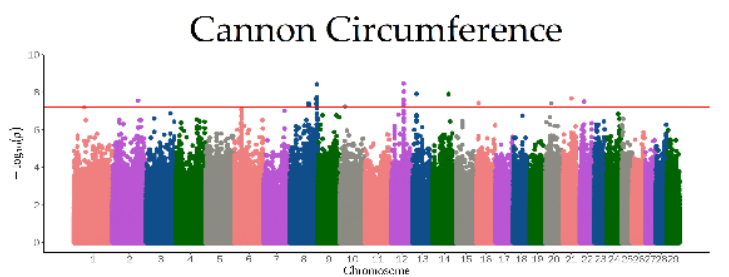

D

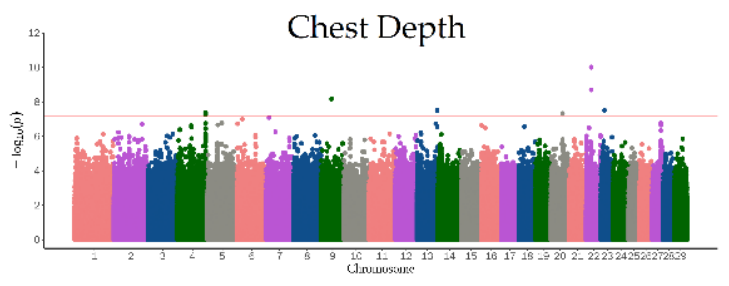

F

Figure 3. Manhattan plots of the BH (A), BL (B), HG (C), CC (D), CW (E), and CD (F) drawn by the observed $p$-value for each SNP. The red horizontal lines in the Manhattan plots show the significance threshold $\left(6.01 \times 10^{-8}\right)$. 


\section{Discussion}

Although thousands of candidate genes underlying economically important traits have been identified in domestic animals, little progress has been made in goat genomics. However, since 2014, low-cost gene chips have had a broad impact on the analysis of the genetic structure of traits of economic interest, as well as on the study of goat population structures at a global scale [32,33]. Luigi-Sierra et al. [8] used the GoatSNP50 BeadChip (Illumina Inc., San Diego, USA) to genotype 825 Murciano-Granadina goats and performed a GWAS. They found that two significant SNPs were associated with the medial suspensory ligament. Based on GoatSNP50 BeadChip, the horn statuses of Boer goats, cashmere goats, and grassland goats were analyzed, revealing a hornless region with a strong selection signal on chromosome 1 [34]. Martin et al. [15] conducted a GWAS of Saanen dairy goats and identified three significant SNPs associated with coat color.

In 2012, a female Yunnan black goat was used to construct a reference genome for the first time by combining Illumina next-generation sequencing and whole-genome mapping techniques [35]. Whole-gene resequencing has become the most rapid and effective method in detecting variation, selection signals, and candidate genes. In Yunshang black goats, GWAS, analyses of runs of homozygosity, and the detection of signatures of selection revealed candidate genes affecting the litter size. Among these candidate genes, some were involved in ovarian function (PPP2R5C, CDC25A, ESR1, RPS26, and SERPINBs); seasonal reproduction (DIO3, BTG1, and CRYM); and metabolism (OSBPL8, SLC39A5, and SERPINBs) [36]. In addition, Yang et al. [37] used whole-genome sequencing data from 46 Australian Boer goats to detect SNPs and identify genomic regions associated with muscle development. Finally, 30 candidate genes (e.g., JAK2, KCNQ1, PDE5A, PDLIM5, and TBX5) directly associated with muscle development were obtained. Wang et al. [38] performed whole-genome resequencing on eight Chinese goat breeds in different regions, with a depth of 9-13×. There were about 10 million SNPs per breed, and Lhx2, FGF9, Wnt2, $M C 1 R$, and FGF5 may be related to cashmere production traits. Along with the decreasing cost of next-generation sequencing, the application of WGS is becoming increasingly popular [39]. In the future, WGS has the potential to overtake gene chips, which is the most popular sequencing tool at present.

There have been several genomic studies of DBGs to date; however, these studies are limited by small sample sizes. Genome-wide selection signatures in 31 DBGs from two groups with differences in fecundity revealed that $L R P 1 B$ and GRID2 showed significantly different patterns of linkage disequilibrium [40]. In addition, E et al. [41] classified $\mathrm{CNVs}$ according to the previous results and reported three differentially expressed genes (LOC108633278, PPP1R12A, and YIPF4) between the high-yield and low-yield groups. There was a $C N V$ in the $C B L B$ gene identified at the individual level. Guan et al. identified some candidate regions based on allele frequency differences in the DBGs and Inner Mongolia cashmere goats. Hormone activity and signaling pathways such as neurohypophyseal hormone activity and adipocytokine signaling pathway [5] may affect reproductive or production traits. In general, additional data are needed to validate the results of these studies of DBGs.

In the GWAS, PSTPIP2, which was located on chromosome 24, was significantly associated with BL. According to Lukens et al. [42] and Yao et al. [43], PSTPIP2 negatively regulates IL-1 $\beta$, which plays an important role in osteomyelitis. This gene is highly expressed in synovial cells, suppresses the inflammatory response, and reduces the number of osteoclasts by inhibiting the function of fibroblastic synovial cells. In addition, PSTPIP2 possesses the ability to control the bone resorption process by regulating the assembly of the podosome [44]. Our GO enrichment analysis also revealed that it is related to the molecular function terms actin binding and cytoskeleton binding (Supplementary Materials Table S4).

In the GWAS of $\mathrm{CD}$, the following candidate genes were identified: C7orf57, EDEM1, SUN3, MANEA, CDKAL1, CDH9, CBLN4, and SOX4. Among these, Chio et al. found an association between C7orf57 and sporadic amyotrophic lateral sclerosis based on 553 pa- 
tients [45]. CDKAL1 was identified as an essential gene for the maintenance of normal mitochondrial morphology and function in adipose tissue [46]. In addition, CDKAL1 affects the transcription of growth hormone genes [47]. SOX4 regulates apoptosis in cardiomyocytes and controls embryonic and cardiovascular development [48]. KEGG and GO enrichment analyses indicated that it is involved in molecular functions, including DNA binding and the mitotic cell cycle, as well as biological processes, such as positive protein regulation and DNA damage detection (Supplementary Materials Tables S4 and S5).

We detected many significant SNPs associated with CC, especially on chromosomes 8 and 12. For example, CCL19 on chromosome 8 promotes ligament ossification [49]. A lack of UBAP1 leads to neurological disorders via the ESCRT pathway, such as hereditary spastic paraplegia [50,51]. Additionally, FGF9 and SGCG, located on chromosome 12, are noteworthy candidates. FGF9 maintains the osteogenic progenitor cell population by the activation of Akt signaling [52,53]. In contrast, SGCG can cause malnutrition and reduce muscle function [54]. Additionally, FIGN, which can affect mammalian development [55], and SIPA1L1, which upregulates osteoblast development via miR-617/smad3 [56], are potential genes involved in the regulation of the development of muscle and bone.

We identified many genetic markers that may affect the growth performance of goats. These significant regions have been reported in other studies, supporting the validity of our results. Information on the genomic regions found in this study can facilitate the identification of candidate genes for body conformation traits. However, we did not detect any significant associations for $\mathrm{CW}$ and $\mathrm{HG}$, and these results may be due to imprecise phenotypic determinations or an inadequate sample size. In addition to technical factors, the polygenic basis of the morphological traits makes it difficult to detect genetic factors with small phenotypic effects. Ultimately, these results provide guidance for genomic selection in the DBG population for highly efficient genetic improvement.

\section{Conclusions}

In this study, genomic and phenotypic data for 150 DBGs were collected, and genomewide association studies were carried out. An analysis revealed that PSTPIP2, C7orf57, CCL19, FGF9, SGCG, FIGN, and SIPA1L likely contribute to the regulation of bone and muscle development. This was the first large-scale genome survey of DBG, and the results are expected to contribute to genomic selection. Furthermore, our approach can be used for the discovery of genetic markers in goats.

Supplementary Materials: The following supporting information can be downloaded at https: / / www.mdpi.com/article/10.3390/ani12050548/s1: Figure S1: The national standard for the body measurements of goats in China. Figure S2: Frequency distribution and test for the normal distribution of six body conformation traits. Figure S3: The kinship relationships of goats. Table S1: The statistics of the production performances of 150 DBGs. Table S2: SNP statistics for all samples. Table S3: Significant SNPs identified by GWAS. Table S4: The enriched GO terms analysis. Table S5: KEGG statistics.

Author Contributions: B.G.: methodology, investigation, visualization, writing-original draft, software, investigation, and validation; R.S.: methodology and investigation; X.F.: investigation; J.Z.: methodology and investigation; Z.Z.: investigation; D.H.: resources; Y.Z. (Yuanping Zhao): investigation; and Y.Z. (Yongju Zhao): supervision and writing-reviewing and editing. All authors have read and agreed to the published version of the manuscript.

Funding: This work was financially supported by the National Natural Science Foundation of China (No.31772564), Key Project of Chongqing Natural Science Foundation (cstc2020jcyj-zdxmX0015), Chongqing's Modern Agricultural Industry Technology System Program for Herbivore (2022), Collection, Utilization, and Innovation of Germplasm Resources by Research Institutes and En-terprises of Chongqing (Cqnyncw-kqlhtxm).

Institutional Review Board Statement: All animal experiments followed the Southwest University Institutional Animal Care and Use Committee regulations (2019, No. GB14925-2010). 
Data Availability Statement: The data presented in this study are available on request from the corresponding author.

Acknowledgments: We appreciate the Chongqing Key Laboratory of Forage and Herbivores and Chongqing Engineering Research Center for providing the experimental farm and technical assistance.

Conflicts of Interest: The authors declare no conflict of interest.

\section{References}

1. Naderi, S.; Rezaei, H.R.; Pompanon, F.; Blum, M.G.B.; Negrini, R.; Naghash, H.R.; Balkiz, O.; Mashkour, M.; Gaggiotti, O.E.; Ajmone-Marsan, P.; et al. The goat domestication process inferred from large-scale mitochondrial DNA analysis of wild and domestic individuals. Proc. Natl. Acad. Sci. USA 2008, 105, 17659-17664. [CrossRef] [PubMed]

2. Dhanda, J.S.; Taylor, D.G.; Murray, P.J.; Pegg, R.B.; Shand, P.J. Goat meat production: Present status and future possibilities. Asian Australas. J. Anim. Sci. 2003, 16, 1842-1852. [CrossRef]

3. Pophiwa, P.; Webb, E.C.; Frylinck, L. A review of factors affecting goat meat quality and mitigating strategies. Small Rumin. Res. 2020, 183, 7. [CrossRef]

4. Wei, C.H.; Lu, J.; Xu, L.Y.; Liu, G.; Wang, Z.G.; Zhao, F.P.; Zhang, L.; Han, X.; Du, L.X.; Liu, C.S. Genetic Structure of Chinese Indigenous Goats and the Special Geographical Structure in the Southwest China as a Geographic Barrier Driving the Fragmentation of a Large Population. PLoS ONE 2014, 9, 12. [CrossRef]

5. Guan, D.; Luo, N.; Tan, X.; Zhao, Z.; Huang, Y.; Na, R.; Zhang, J.; Zhao, Y. Scanning of selection signature provides a glimpse into important economic traits in goats (Capra hircus). Sci. Rep. 2016, 6, 36372. [CrossRef]

6. Cam, M.A.; Olfaz, M.; Soydan, E. Body Measurements Reflect Body Weights and Carcass Yields in Karayaka Sheep. Asian J. Anim. Vet. Adv. 2010, 5, 120-127. [CrossRef]

7. Yoshida, G.M.; Yanez, J.M. Multi-trait GWAS using imputed high-density genotypes from whole-genome sequencing identifies genes associated with body traits in Nile tilapia. BMC Genom. 2021, 22, 57. [CrossRef]

8. Luigi-Sierra, M.G.; Landi, V.; Guan, D.; Delgado, J.V.; Castello, A.; Cabrera, B.; Marmol-Sanchez, E.; Alvarez, J.F.; GomezCarpio, M.; Martinez, A.; et al. A genome-wide association analysis for body, udder, and leg conformation traits recorded in Murciano-Granadina goats. J. Dairy Sci. 2020, 103, 11605-11617. [CrossRef]

9. Wang, Z.; Wang, C.; Guo, Y.; She, S.; Wang, B.; Jiang, Y.; Bai, Y.; Song, X.; Li, L.; Shi, L.; et al. Screening of Deletion Variants within the Goat PRDM6 Gene and Its Effects on Growth Traits. Animals 2020, 10, 208. [CrossRef]

10. Liu, X.; Ma, L.; Wang, M.; Wang, K.; Li, J.; Yan, H.; Zhu, H.; Lan, X. Two indel variants of prolactin receptor (PRLR) gene are associated with growth traits in goat. Anim. Biotechnol. 2020, 31, 314-323. [CrossRef]

11. Hirschhorn, J.N.; Daly, M.J. Genome-wide association studies for common diseases and complex traits. Nat. Rev. Genet. 2005, 6, 95-108. [CrossRef] [PubMed]

12. Bai, Y.Y.; Li, J.; Zhu, H.J.; Liu, J.W.; Dong, S.W.; Li, L.P.; Qu, L.; Chen, H.; Song, X.Y.; Lan, X.Y. Deletion mutation within the goat PPP3CA gene identified by GWAS significantly affects litter size. Reprod. Fertil. Dev. 2021, 33, 476-483. [CrossRef] [PubMed]

13. Islam, R.; Liu, X.X.; Gebreselassie, G.R.M.; Abied, A.D.; Ma, Q.; Ma, Y.H. Genome-wide association analysis reveals the genetic locus for high reproduction trait in Chinese Arbas Cashmere goat. Genes Genom. 2020, 42, 893-899. [CrossRef]

14. Guo, J.Z.; Jiang, R.; Mao, A.; Liu, G.E.; Zhan, S.Y.; Li, L.; Zhong, T.; Wang, L.J.; Cao, J.X.; Chen, Y.; et al. Genome-wide association study reveals 14 new SNPs and confirms two structural variants highly associated with the horned/polled phenotype in goats. BMC Genom. 2021, 22, 10. [CrossRef] [PubMed]

15. Martin, P.M.; Palhiere, I.; Ricard, A.; Tosser-Klopp, G.; Rupp, R. Genome Wide Association Study Identifies New Loci Associated with Undesired Coat Color Phenotypes in Saanen Goats. PLoS ONE 2016, 11, 15. [CrossRef] [PubMed]

16. Tilahun, Y.; Gipson, T.A.; Alexander, T.; McCallum, M.L.; Hoyt, P.R. Genome-Wide Association Study towards Genomic Predictive Power for High Production and Quality of Milk in American Alpine Goats. Int. J. Genom. 2020, 2020, 12. [CrossRef]

17. Scholtens, M.; Jiang, A.; Smith, A.; Littlejohn, M.; Lehnert, K.; Snell, R.; Lopez-Villalobos, N.; Garrick, D.; Blair, H. Genome-wide association studies of lactation yields of milk, fat, protein and somatic cell score in New Zealand dairy goats. J. Anim. Sci. Biotechnol. 2020, 11, 55. [CrossRef]

18. Wang, K.; Liu, X.; Qi, T.; Hui, Y.; Yan, H.; Qu, L.; Lan, X.; Pan, C. Whole-genome sequencing to identify candidate genes for litter size and to uncover the variant function in goats (Capra hircus). Genomics 2021, 113 Pt 1, 142-150. [CrossRef]

19. Wu, P.X.; Wang, K.; Zhou, J.; Chen, D.J.; Yang, Q.; Yang, X.D.; Liu, Y.H.; Feng, B.; Jiang, A.N.; Shen, L.Y.; et al. GWAS on Imputed Whole-Genome Resequencing From Genotyping-by-Sequencing Data for Farrowing Interval of Different Parities in Pigs. Front. Genet. 2019, 10, 1012. [CrossRef]

20. Sanchez, M.P.; Guatteo, R.; Davergne, A.; Saout, J.; Grohs, C.; Deloche, M.C.; Taussat, S.; Fritz, S.; Boussaha, M.; Blanquefort, P.; et al. Identification of the ABCC4, IER3, and CBFA2T2 candidate genes for resistance to paratuberculosis from sequence-based GWAS in Holstein and Normande dairy cattle. Genet. Sel. Evol. 2020, 52, 17. [CrossRef]

21. Tribout, T.; Croiseau, P.; Lefebvre, R.; Barbat, A.; Boussaha, M.; Fritz, S.; Boichard, D.; Hoze, C.; Sanchez, M.P. Confirmed effects of candidate variants for milk production, udder health, and udder morphology in dairy cattle. Genet. Sel. Evol. 2020, 52, 1-26. [CrossRef] [PubMed] 
22. van den Berg, S.; Vandenplas, J.; van Eeuwijk, F.A.; Bouwman, A.C.; Lopes, M.S.; Veerkamp, R.F. Imputation to whole-genome sequence using multiple pig populations and its use in genome-wide association studies. Genet. Sel. Evol. 2019, 51, 1-13. [CrossRef] [PubMed]

23. Talouarn, E.; Bardou, P.; Palhiere, I.; Oget, C.; Clement, V.; VarGoats, C.; Tosser-Klopp, G.; Rupp, R.; Robert-Granie, C. Genome wide association analysis on semen volume and milk yield using different strategies of imputation to whole genome sequence in French dairy goats. BMC Genet. 2020, 21, 19. [CrossRef]

24. Bickhart, D.M.; Rosen, B.D.; Koren, S.; Sayre, B.L.; Hastie, A.R.; Chan, S.; Lee, J.; Lam, E.T.; Liachko, I.; Sullivan, S.T.; et al. Single-molecule sequencing and chromatin conformation capture enable de novo reference assembly of the domestic goat genome. Nat. Genet. 2017, 49, 643-650. [CrossRef] [PubMed]

25. Li, H.; Durbin, R. Fast and accurate short read alignment with Burrows-Wheeler transform. Bioinformatics 2009, 25, 1754-1760. [CrossRef] [PubMed]

26. McKenna, A.; Hanna, M.; Banks, E.; Sivachenko, A.; Cibulskis, K.; Kernytsky, A.; Garimella, K.; Altshuler, D.; Gabriel, S.; Daly, M.; et al. The Genome Analysis Toolkit: A MapReduce framework for analyzing next-generation DNA sequencing data. Genome Res. 2010, 20, 1297-1303. [CrossRef] [PubMed]

27. Le, S.; Josse, J.; Husson, F. FactoMineR: An R package for multivariate analysis. J. Stat. Softw. 2008, 25, 1-18. [CrossRef]

28. Browning, B.L.; Zhou, Y.; Browning, S.R. A One-Penny Imputed Genome from Next-Generation Reference Panels. Am. J. Hum. Genet. 2018, 103, 338-348. [CrossRef]

29. Yu, J.; Pressoir, G.; Briggs, W.H.; Vroh Bi, I.; Yamasaki, M.; Doebley, J.F.; McMullen, M.D.; Gaut, B.S.; Nielsen, D.M.; Holland, J.B.; et al. A unified mixed-model method for association mapping that accounts for multiple levels of relatedness. Nat. Genet. 2006, 38, 203-208. [CrossRef]

30. Zhou, X.; Stephens, M. Genome-wide efficient mixed-model analysis for association studies. Nat. Genet. 2012, 44, 821-824. [CrossRef]

31. Yu, G.; Wang, L.G.; Han, Y.; He, Q.Y. clusterProfiler: An R package for comparing biological themes among gene clusters. OMICS 2012, 16, 284-287. [CrossRef] [PubMed]

32. Bertolini, F.; Servin, B.; Talenti, A.; Rochat, E.; Kim, E.S.; Oget, C.; Palhiere, I.; Crisa, A.; Catillo, G.; Steri, R.; et al. Signatures of selection and environmental adaptation across the goat genome post-domestication. Genet. Sel. Evol. 2018, 50, 57. [CrossRef] [PubMed]

33. Colli, L.; Milanesi, M.; Talenti, A.; Bertolini, F.; Chen, M.H.; Crisa, A.; Daly, K.G.; Del Corvo, M.; Guldbrandtsen, B.; Lenstra, J.A.; et al. Genome-wide SNP profiling of worldwide goat populations reveals strong partitioning of diversity and highlights post-domestication migration routes. Genet. Sel. Evol. 2018, 50,1-20. [CrossRef] [PubMed]

34. Kijas, J.W.; Ortiz, J.S.; McCulloch, R.; James, A.; Brice, B.; Swain, B.; Tosser-Klopp, G.; the International Goat Genome Consortium Genetic diversity and investigation of polledness in divergent goat populations using 52088 SNPs. Anim. Genet. 2013, 44, 325-335. [CrossRef] [PubMed]

35. Dong, Y.; Xie, M.; Jiang, Y.; Xiao, N.Q.; Du, X.Y.; Zhang, W.G.; Tosser-Klopp, G.; Wang, J.H.; Yang, S.; Liang, J.; et al. Sequencing and automated whole-genome optical mapping of the genome of a domestic goat (Capra hircus). Nat. Biotechnol. 2013, 31, 135-141. [CrossRef]

36. Tao, L.; He, X.Y.; Jiang, Y.T.; Lan, R.; Li, M.; Li, Z.M.; Yang, W.F.; Hong, Q.H.; Chu, M.X. Combined approaches to reveal genes associated with litter size in Yunshang black goats. Anim. Genet. 2020, 51, 924-934. [CrossRef]

37. Yang, B.G.; Yuan, Y.; Zhou, D.K.; Ma, Y.H.; Mahrous, K.F.; Wang, S.Z.; He, Y.M.; Duan, X.H.; Zhang, W.Y.; E, G. Genome-wide selection signal analysis of Australian Boer goat reveals artificial selection imprinting on candidate genes related to muscle development. Anim. Genet. 2021, 52, 550-555. [CrossRef]

38. Li, X.K.; Su, R.; Wan, W.T.; Zhang, W.G.; Jiang, H.Z.; Qiao, X.; Fan, Y.X.; Zhang, Y.J.; Wang, R.J.; Liu, Z.H.; et al. Identification of selection signals by large-scale whole-genome resequencing of cashmere goats. Sci. Rep. 2017, 7, 1-10. [CrossRef]

39. Visscher, P.M.; Wray, N.R.; Zhang, Q.; Sklar, P.; McCarthy, M.I.; Brown, M.A.; Yang, J. 10 Years of GWAS Discovery: Biology, Function, and Translation. Am. J. Hum. Genet. 2017, 101, 5-22. [CrossRef]

40. E, G.X.; Duan, X.H.; Zhang, J.H.; Huang, Y.F.; Zhao, Y.J.; Na, R.S.; Zhao, Z.Q.; Ma, Y.H.; Chu, M.X.; Basang, W.D.; et al. Genomewide selection signatures analysis of litter size in Dazu black goats using single-nucleotide polymorphism. 3 Biotech $2019,9,336$. [CrossRef]

41. E, G.-X.; Zhu, Y.B.; Basang, W.D.; Na, R.S.; Han, Y.G.; Zeng, Y. Comparative and selection sweep analysis of CNV was associated to litter size in Dazu black goats. Anim. Biotechnol. 2021, 32, 792-797. [CrossRef] [PubMed]

42. Lukens, J.R.; Gross, J.M.; Calabrese, C.; Iwakura, Y.; Lamkanfi, M.; Vogel, P.; Kanneganti, T.D. Critical role for inflammasomeindependent IL-1beta production in osteomyelitis. Proc. Natl. Acad. Sci. USA 2014, 111, 1066-1071. [CrossRef] [PubMed]

43. Yao, Y.; Cai, X.; Yu, H.; Xu, Q.; Li, X.; Yang, Y.; Meng, X.; Huang, C.; Li, J. PSTPIP2 attenuates joint damage and suppresses inflammation in adjuvant-induced arthritis. Eur. J. Pharmacol. 2019, 859, 172558. [CrossRef] [PubMed]

44. Sztacho, M.; Segeletz, S.; Sanchez-Fernandez, M.A.; Czupalla, C.; Niehage, C.; Hoflack, B. BAR Proteins PSTPIP1/2 Regulate Podosome Dynamics and the Resorption Activity of Osteoclasts. PLoS ONE 2016, 11, e0164829. [CrossRef] [PubMed]

45. Chio, A.; Schymick, J.C.; Restagno, G.; Scholz, S.W.; Lombardo, F.; Lai, S.L.; Mora, G.; Fung, H.C.; Britton, A.; Arepalli, S.; et al. A two-stage genome-wide association study of sporadic amyotrophic lateral sclerosis. Hum. Mol. Genet. 2009, 18, 1524-1532. [CrossRef] [PubMed] 
46. Palmer, C.J.; Bruckner, R.J.; Paulo, J.A.; Kazak, L.; Long, J.Z.; Mina, A.I.; Deng, Z.; LeClair, K.B.; Hall, J.A.; Hong, S.; et al. Cdkal1, a type 2 diabetes susceptibility gene, regulates mitochondrial function in adipose tissue. Mol. Metab. 2017, 6, 1212-1225. [CrossRef]

47. Takesue, Y.; Wei, F.Y.; Fukuda, H.; Tanoue, Y.; Yamamoto, T.; Chujo, T.; Shinojima, N.; Yano, S.; Morioka, M.; Mukasa, A.; et al. Regulation of growth hormone biosynthesis by Cdk5 regulatory subunit associated protein 1-like 1 (CDKAL1) in pituitary adenomas. Endocr. J. 2019, 66, 807-816. [CrossRef]

48. Zhang, L.; Lv, L.; Zheng, N.; Li, R.; Yang, R.; Li, T.; Li, Y.; Liu, Y.; Luo, H.; Li, X.; et al. Suppression of Sox4 protects against myocardial ischemic injury by reduction of cardiac apoptosis in mice. J. Cell. Physiol. 2021, 236, 1094-1104. [CrossRef]

49. Qin, Y.; He, L.D.; Sheng, Z.J.; Yong, M.M.; Sheng, Y.S.; Dong, X.W.; Wen, T.W.; Ming, Z.Y. Increased CCL19 and CCL21 levels promote fibroblast ossification in ankylosing spondylitis hip ligament tissue. BMC Musculoskelet. Disord. 2014, 15, 316. [CrossRef]

50. Nan, H.; Ichinose, Y.; Tanaka, M.; Koh, K.; Ishiura, H.; Mitsui, J.; Mizukami, H.; Morimoto, M.; Hamada, S.; Ohtsuka, T.; et al UBAP1 mutations cause juvenile-onset hereditary spastic paraplegias (SPG80) and impair UBAP1 targeting to endosomes. J. Hum. Genet. 2019, 64, 1055-1065. [CrossRef]

51. Wang, J.; Hou, Y.; Qi, L.; Zhai, S.; Zheng, L.; Han, L.; Guo, Y.; Zhang, B.; Miao, P.; Lou, Y.; et al. Autosomal dominant hereditary spastic paraplegia caused by mutation of UBAP1. Neurogenetics 2020, 21, 169-177. [CrossRef] [PubMed]

52. Gao, X.; Yao, X.; Yang, H.; Deng, K.; Guo, Y.; Zhang, T.; Zhang, G.; Wang, F. Role of FGF9 in sheep testis steroidogenesis during sexual maturation. Anim. Reprod. Sci. 2018, 197, 177-184. [CrossRef] [PubMed]

53. Wang, L.; Roth, T.; Abbott, M.; Ho, L.; Wattanachanya, L.; Nissenson, R.A. Osteoblast-derived FGF9 regulates skeletal homeostasis. Bone 2017, 98, 18-25. [CrossRef] [PubMed]

54. Demonbreun, A.R.; Wyatt, E.J.; Fallon, K.S.; Oosterbaan, C.C.; Page, P.G.; Hadhazy, M.; Quattrocelli, M.; Barefield, D.Y.; McNally, E.M. A gene-edited mouse model of limb-girdle muscular dystrophy 2C for testing exon skipping. Dis. Model. Mech. 2019, 13, dmm040832. [CrossRef]

55. Cox, G.A.; Mahaffey, C.L.; Nystuen, A.; Letts, V.A.; Frankel, W.N. The mouse fidgetin gene defines a new role for AAA family proteins in mammalian development. Nat. Genet. 2000, 26, 198-202. [CrossRef]

56. Ge, X.; Li, Z.; Zhou, Z.; Xia, Y.; Bian, M.; Yu, J. Circular RNA SIPA1L1 promotes osteogenesis via regulating the miR-617/Smad3 axis in dental pulp stem cells. Stem. Cell Res. Ther. 2020, 11, 364. [CrossRef] 